\title{
Discrepâncias na correlação de exames colposcópicos, citológicos e histológicos: estudo de caso
}

Sousa $M^{1}+$, Gonçalves $M^{2}+$ e Pinto $A^{3}+$

\author{
${ }^{1}$ Microdiag, Laboratório de Anatomia Patológica, Lda. - Leiria, Portugal \\ ${ }^{2}$ Centro Hospitalar de Leiria - Leiria, Portugal \\ ${ }^{3}$ Laboratório Dr. Albino Oliveira - LAP - Santa Maria da Feira, Portugal \\ † Estes autores contribuíram de igual modo na realização do trabalho \\ Recebido: novembro 2018/ Publicado: dezembro 2018 \\ https://doi.org/10.26537/citotech.v0i4.3012
}

*Autor correspondente:

Mariana Sousa

marianasousa30@gmail.com

\section{RESUMO}

\section{Objetivo}

O presente artigo pretende expor um caso de ausência de correlação entre os exames colposcópico, citológico e histológico.

\section{Apresentação do caso}

Numa consulta de ginecologia de rotina, a paciente, de 54 anos, realizou uma citologia ginecológica em meio líquido, cuja observação microscópica resultou no diagnóstico de carcinoma epidermóide. Posteriormente, a paciente foi encaminhada para uma sequência de consultas de followup, tendo realizado uma biópsia do colo que demonstrou marcada atrofia, sendo negativa para lesão intraepitelial; uma pesquisa e tipificação do vírus do Papiloma Humano positiva para os genótipos 35 e 56 (alto risco); e uma conização, da qual resultou um diagnóstico de lesão intraepitelial pavimentosa de baixo grau.

\section{Discussão}

Após a realização de pesquisa bibliográfica que relatasse exemplos de casos de discrepância entre a colposcopia, a citologia e a histologia, verificou-se que os falsos negativos, tanto na colposcopia, como nos exames histológicos, bem como a sobrevalorização do diagnóstico citológico, poderão ser hipóteses causais para a disparidade de resultados apresentados neste caso.

\section{Conclusão}

Este estudo remete para a importância da correlação entre a citologia e a histologia, assim como a necessidade de comunicação entre os vários profissionais de saúde das diversas áreas envolvidas.

Palavras-chave: Correlação; Citologia; Histologia; Carcinoma epidermóide; Atrofia. 


\section{INTRODUÇÃO}

O cancro do colo do útero (CCU) é a segunda neoplasia mais frequente nas mulheres a nível mundial, seguido do cancro da mama. Em Portugal, é o oitavo cancro mais frequente nas mulheres ${ }^{1}$.

A infeção pelo HPV, do inglês Human Papillomavirus, é apontada como o principal fator etiológico de $\mathrm{CCU}$, nomeadamente as infeções pelos genótipos de alto risco, tais como o $16,18,35$ e $56^{2}$. Sendo o HPV um vírus sexualmente transmissível, a sua incidência é alta ${ }^{3}$.

A maioria dos CCU corresponde a carcinomas epidermóides, que na maior parte dos casos, são precedidos por lesões epiteliais precursoras, que se dividem em lesão intraepitelial de baixo grau (LSIL, do inglês Low Squamous Intraepithelial Lesion/CIN1, do inglês Cervical Intraepithelial Neoplasia) e lesão intraepitelial de alto grau (HSIL, do inglês High Squamous Intraepithelial Lesion/CIN2,3) ${ }^{4}$. Estas lesões precursoras podem ser detetadas através da realização do exame citológico (teste de Papanicolaou), através de uma colposcopia em consultas de rotina ginecológica ou através de programas de rastreio, sendo posteriormente confirmadas pela histologia ${ }^{5}$.

A correlação entre o estudo histológico e o citológico visa aumentar a sensibilidade e especificidade do diagnóstico final ${ }^{6}$. Quando não existe correlação, é frequente assumir-se que a discrepância se deve a um erro no screening, não sendo de excluir a hipótese de outros fatores como causa das discrepâncias (má amostragem, erros de processamento e erros de interpretação diagnóstica na histologia) ${ }^{7}$.

No presente estudo, pretendeu-se expor um caso de discordância diagnóstica entre a colposcopia, a citologia e a histologia.

\section{APRESENTAÇÃO DO CASO}

Mulher de 54 anos, menopáusica, com antecedentes de inflamação persistente, consultou o seu ginecologista e efetuou colposcopia, que revelou colo sem lesões, tendo sido colhido material para citologia em meio líquido (método Cellprep®).

A lâmina de citologia foi corada pela técnica de Papanicolaou, tendo a análise microscópica revelado um esfregaço satisfatório, com representação da zona de transformação, onde se verificou a presença de pequenos grupos de células pavimentosas com atrofia marcada. Verificou-se ainda a presença de células com aumento da relação núcleo/citoplasma, pleomórficas, algumas queratinizadas, alternadas com outras células de citoplasma escasso e denso 8,9 (Fig.1).

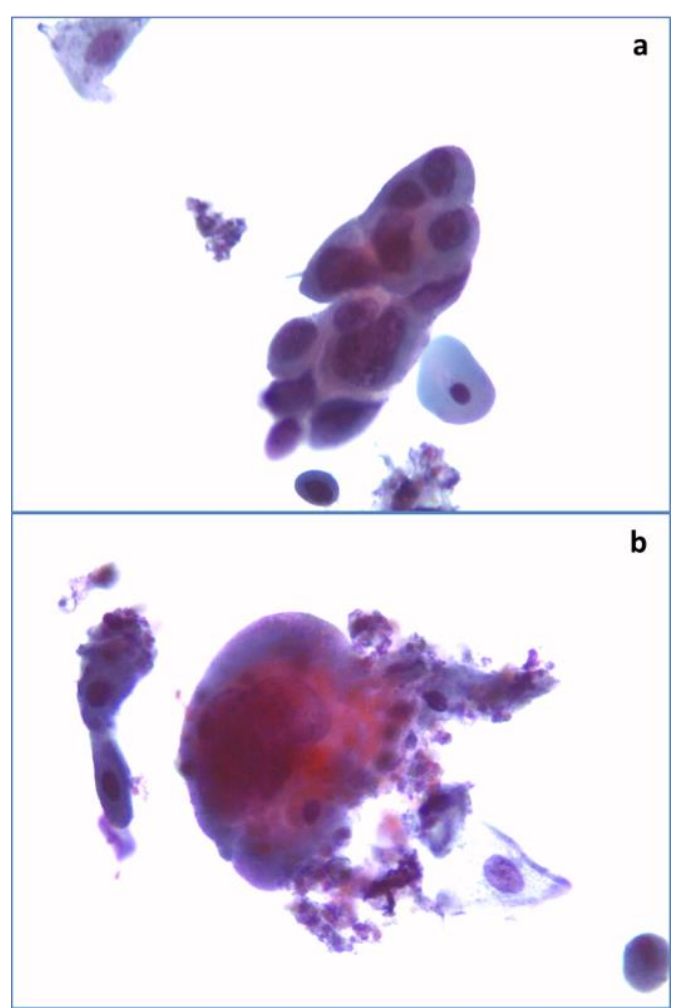

Fig.1 - a) Grupo de células imaturas, com relação núcleo/citoplasma aumentada, citoplasma denso, anisocariose e hipercromasia nuclear; b) Célula isolada, com citoplasma denso, núcleo muito aumentado, com membrana irregular. Citologia de base líquida - Cellprep ${ }^{\circledR}$; coloração de Papanicolaou, 400x. 
Os núcleos apresentavam-se muito aumentados, hipercromáticos, com irregularidade da membrana e anisocariose acentuada. Estes exibiam uma cromatina grosseira, irregular e mal distribuída, por vezes, com nucléolo proeminente 8,9 (Fig.2). O fundo apresentava-se sujo e algo hemático, sendo sugestivo de diátese tumoral (Fig.3).

Tendo em conta as características acima descritas, o diagnóstico foi carcinoma epidermóide.

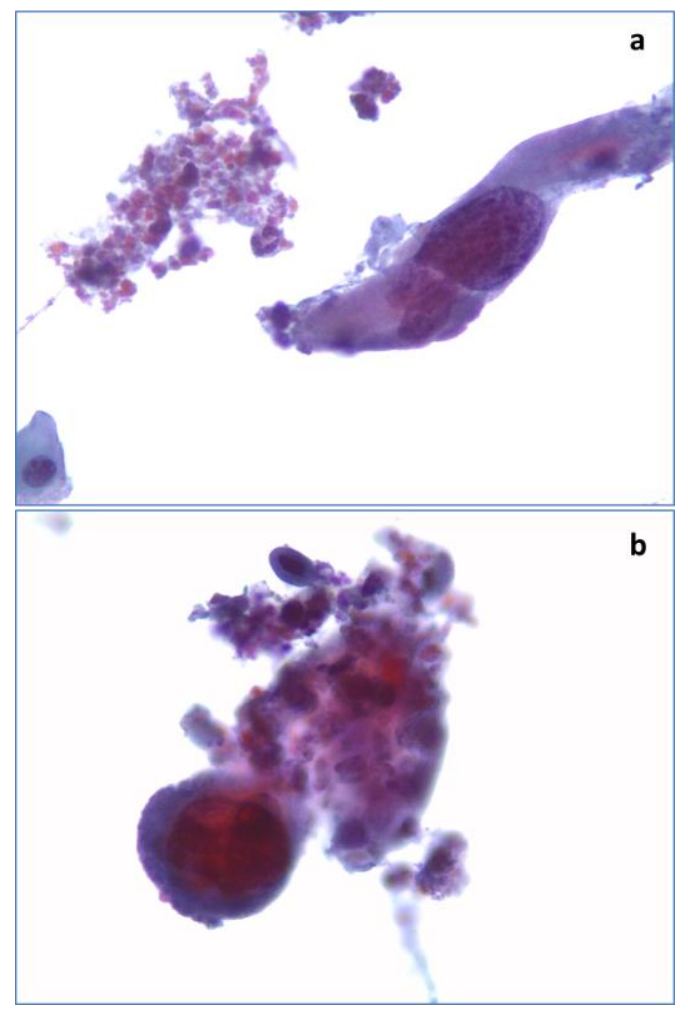

Fig.2 - a) Célula isolada, de citoplasma denso alongado, com núcleo muito aumentado, com membrana irregular, cromatina grosseira e irregularmente distribuída; b) Célula isolada, com citoplasma denso, com relação núcleo/citoplasma aumentada e núcleo hipercromático. Citologia de base líquida - Cellprep®; coloração de Papanicolaou, 400x.

\section{FOLLOW UP}

Após o resultado citológico, a paciente foi submetida a exame colposcópico, com curetagem do endocolo e colheita de biópsias do colo. Para além disso, foi realizada nova colheita citológica para deteção e tipificação de HPV, tendo a mesma sido positiva para HPV 35 e 56 (alto risco). Não tendo o estudo colposcópico revelado alterações visíveis, e tendo em conta a proposta diagnóstica avançada pela citologia, foi solicitada a revisão da lâmina. O diagnóstico manteve-se inalterado.

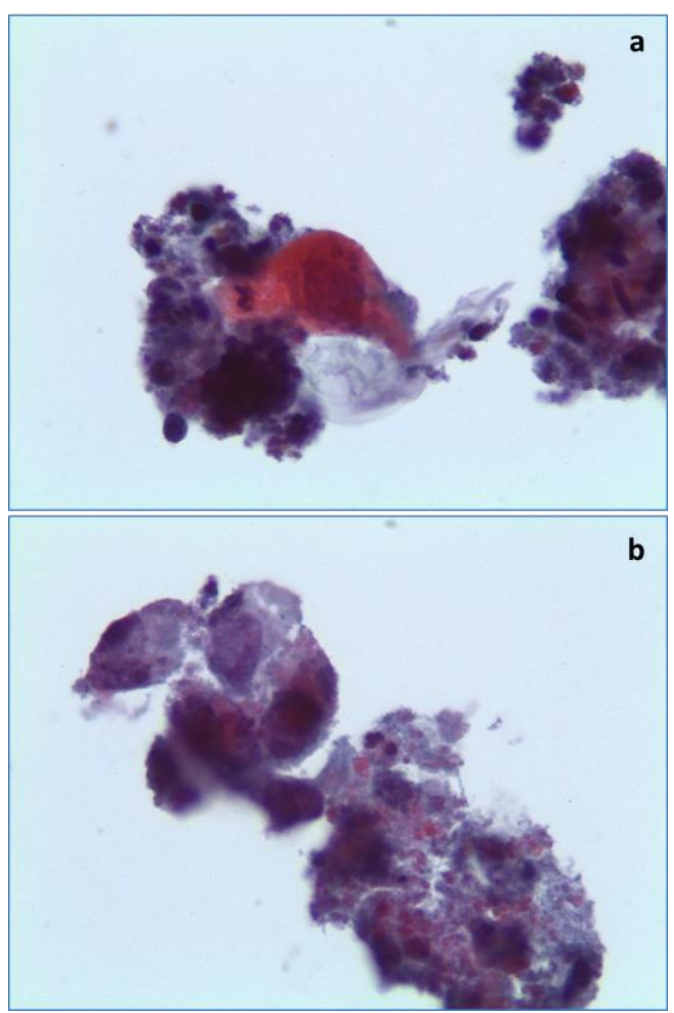

Fig.3 - a) Célula isolada, com citoplasma alongado, denso e queratinizado, núcleo hipercromático, em fundo sujo com detritos; b) Grupo de células imaturas, com relação núcleo/citoplasma aumentada, anisocariose e hipercromasia nuclear, em fundo de detritos, sugestivo de diátese tumoral. Citologia de base líquida - Cellprep®; coloração de Papanicolaou, 400x.

O novo exame citológico revelou 0 mesmo tipo de achados que o primeiro, tendo o diagnóstico sido HSIL com achados sugestivos de carcinoma. Foi igualmente sugerida a realização de repetição após terapêutica estrogénica.

O estudo histológico do produto de curetagem e das biópsias de colo revelou discrepâncias entre a citologia e a histologia, uma vez que a biopsia não revelou alterações, 
apresentando achados celulares normais, não sendo observados sinais tradutores de lesão intraepitelial pavimentosa (Fig.4a e 4b).

Posteriormente, a utente foi submetida a uma conização com ansa diatérmica, assim como uma nova citologia de endocolo.

O estudo citológico do canal endocervical visou descobrir eventuais focos de lesão com localização endocervical. No entanto, o resultado foi negativo para lesão intraepitelial ou neoplasia maligna.

O estudo da conização revelou a presença de LSIL, com preenchimento de recessos endocervicais, presença de mitoses, zonas atípicas com lesão intraepitelial pavimentosa não graduável e áreas de necrose, não tendo sido confirmado, ainda assim, o estadio apontado pela citologia (Fig.4c e 4d).

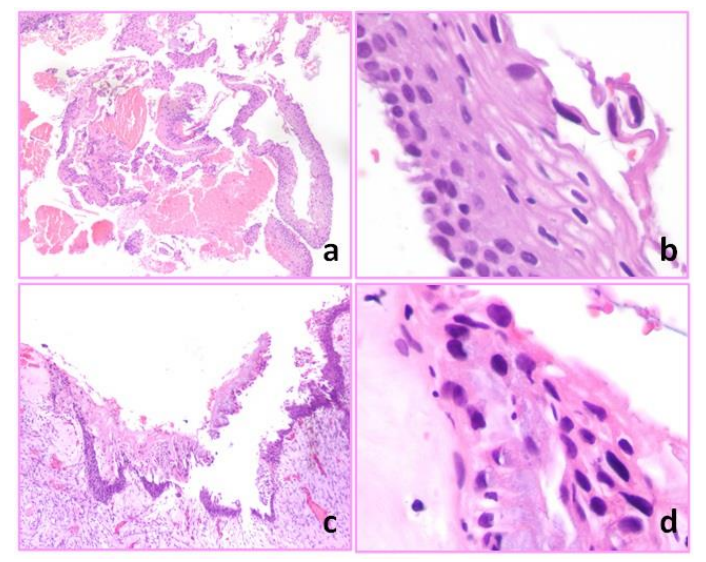

Fig.4- a) Biópsia do colo, negativa para lesão intraepitelial; b) Biópsia do colo, negativa para lesão intraepitelial, no entanto, com células malpighianas soltas, queratinizadas, com núcleo hipercromático e aumento de volume; c) Fragmento de conização com área de "descolamento" do epitélio; d) Fragmento de conização com lesão pavimentosa intraepitelial de baixo grau e atipia $\mathrm{CIN}$ não graduável. Coloração de Hematoxilina-Eosina; 100x $(\mathbf{a}, \mathbf{c}) ; 400 x(\mathbf{b}, \mathbf{d})$.

Para além disso, o estudo das margens cirúrgicas revelou que as mesmas estavam livres de lesão, à exceção da margem exocervical que interessava áreas de LSIL.

Após ter sido sujeita a terapêutica hormonal, a utente fez nova colheita citológica e histológica. A avaliação da lâmina de citologia resultou num diagnóstico de LSIL, tendo o resultado da biópsia coincidido com este diagnóstico (Tabela 1).

Tabela 1. Resumo dos exames realizados pela paciente e respetivos resultados

\begin{tabular}{|c|c|c|}
\hline Data & Exame efetuado & Resultado \\
\hline Dezembro 2016 & $\begin{array}{l}\text { Citologia cervico- } \\
\text { vaginal }\end{array}$ & Carcinoma epidermóide \\
\hline \multirow{4}{*}{$\begin{array}{l}\text { Dezembro } 2016 \\
\text { (1 semana após) }\end{array}$} & Colposcopia & $\begin{array}{l}\text { Colo muito atrófico, sem outras } \\
\text { alterações visíveis }\end{array}$ \\
\hline & $\begin{array}{l}\text { Citologia cervico- } \\
\text { vaginal }\end{array}$ & $\begin{array}{l}\text { HSIL (Obs: Marcada atrofia, } \\
\text { sugestivo de carcinoma, sugere- } \\
\text { se confirmaçãa histológica e } \\
\text { repetição após terapêutica } \\
\text { estrogénica }\end{array}$ \\
\hline & $\begin{array}{l}\text { Pesquisa e } \\
\text { tipificação de } \\
\text { vírus HPV }\end{array}$ & Positivo para HPV 35 e 56 (AR) \\
\hline & $\begin{array}{l}\text { Biópsia do colo e } \\
\text { curetagem } \\
\text { endocervical }\end{array}$ & $\begin{array}{l}\text { Endocolo - Negativo para lesão } \\
\text { escamosa intraepitelial } \\
\text { Colo - Negativo para lesão } \\
\text { escamosa intraepitelial }\end{array}$ \\
\hline \multirow[b]{2}{*}{ Janeiro 2017} & $\begin{array}{l}\text { Citologia do canal } \\
\text { endocervical }\end{array}$ & NILM \\
\hline & $\begin{array}{l}\text { Conização com } \\
\text { ansa diatérmica }\end{array}$ & $\begin{array}{l}\text { Áreas com lesão intraepitelial de } \\
\text { baixo grau e áreas de CIN não } \\
\text { graduável. Margem cirúrgica } \\
\text { exocervical interessando áreas } \\
\text { com lesão de baixo grau }\end{array}$ \\
\hline \multirow[t]{2}{*}{ Maio 2017} & $\begin{array}{l}\text { Citologia cervico- } \\
\text { vaginal (após } \\
\text { terapêutica } \\
\text { estrogénica) }\end{array}$ & LSIL \\
\hline & Biópsia do colo & Lesão intraepitelial de baixo grau \\
\hline
\end{tabular}

\section{DISCUSSÃO DO CASO}

Falsos negativos em exames colposcópicos e histológicos

No caso em estudo, foram verificadas discrepâncias na correlação entre os exames colposcópico, citológico e histológico. Estas discrepâncias, bem como as várias hipóteses de justificação associadas, têm sido alvo de diversos estudos (Tabela 2).

O facto do exame colposcópico ter uma sensibilidade superior, mas especificidade inferior relativamente ao exame citológico, faz com que estes dois procedimentos sejam complementares um do outro, de forma a direcionar o exame histológico e alcançar um diagnóstico mais fiável ${ }^{18}$.

No caso em estudo, a doente realizou uma colposcopia, que revelou um colo muito atrófico sem lesões, não sendo concordante com o diagnóstico citológico. Tal facto pode dever-se a um falso negativo na colposcopia ou a uma sobrevalorização citológica. 
Uma correta avaliação colposcópica permite a localização das zonas de aspeto anormal, representativas da lesão a ser colhida por biopsia. Por vezes, as lesões apresentam localizações ou pequenas dimensões que não são visíveis na colposcopia (por exemplo, lesões na porção inferior do canal endocervical), levando assim a um diagnóstico falso negativo colposcópico (e consequente amostragem inadequada). No caso em estudo, o facto de a colposcopia apresentar um colo sem lesões, não significou que a lesão fosse inexistente $e^{10,17}$.

Tabela 2. Estudos que demonstram a inexistência de correlação colposcópica, citológica e histológica

\begin{tabular}{|c|c|c|c|c|c|c|}
\hline \multirow{2}{*}{ Fonte } & \multicolumn{2}{|c|}{$\begin{array}{l}\text { Diagnóstico } \\
\text { citológico } \\
\text { prévio }\end{array}$} & \multirow{2}{*}{$\begin{array}{c}\begin{array}{c}\text { Estudo } \\
\text { colposcópico }\end{array} \\
\text { Normal }\end{array}$} & \multicolumn{3}{|c|}{$\begin{array}{c}\text { Diagnóstico histológico } \\
\text { posterior }\end{array}$} \\
\hline & $\begin{array}{l}\text { Total } \\
\text { HSIL }\end{array}$ & $\begin{array}{c}\text { Total } \\
\mathrm{Ca}\end{array}$ & & Benigno & CIN 1 & $\begin{array}{l}\text { CIN } \\
2,3\end{array}$ \\
\hline $\begin{array}{l}\text { Joseph et } \\
\text { al., } 1991\end{array}$ & 261 & - & - & 15 & 58 & - \\
\hline \multirow{2}{*}{$\begin{array}{l}\text { Dodd et al., } \\
1992\end{array}$} & 51 & - & - & 14 & 7 & - \\
\hline & - & 1 & - & 1 & - & - \\
\hline $\begin{array}{l}\text { DiBonito et } \\
\text { al., } 1993\end{array}$ & 83 & - & - & 9 & 11 & - \\
\hline $\begin{array}{l}\text { Heatley et } \\
\text { al., } 1998\end{array}$ & 34 & - & - & - & 1 & - \\
\hline \multirow{2}{*}{$\begin{array}{l}\text { Lonky et al., } \\
1999\end{array}$} & 260 & - & - & 79 & 51 & - \\
\hline & - & 3 & - & 0 & 1 & 2 \\
\hline $\begin{array}{l}\text { Szurkus et } \\
\text { al., } 2003\end{array}$ & - & - & 58 & - & - & 34 \\
\hline $\begin{array}{l}\text { Saha et al., } \\
2005\end{array}$ & 9 & - & - & 3 & 1 & - \\
\hline $\begin{array}{l}\text { Kabaca et } \\
\text { al., } 2013\end{array}$ & 61 & - & - & 7 & 11 & - \\
\hline
\end{tabular}

Nota: Na tabela, encontram-se apenas referidos o número de casos cujas discrepâncias se assemelham ao caso em estudo $^{10-17}$

Por outro lado, uma discrepância entre um diagnóstico citológico e um diagnóstico histológico, também se pode dever a falsos negativos na histologia. A maior causa de falsos negativos nas biópsias é, pois, uma colheita inadequada. Os falsos negativos ocorrem em cerca de 1/3 dos diagnósticos histológicos a partir de biópsias de colo uterino $^{13}$. Os diagnósticos citológicos só podem ser verdadeiramente validados através do estudo histológico da conização, em que todos os graus da lesão intraepitelial existentes são devidamente avaliados. Assim, a citologia e a histologia surgem como estudos complementares ${ }^{13}$.
No caso em estudo, a avaliação histológica das biópsias falhou na identificação da lesão, tendo a mesma sido identificada posteriormente na conização. Este aspeto está em concordância com outros estudos que revelaram que colheitas histológicas, realizadas posteriormente a uma biópsia falsa negativa, tendem a demonstrar a lesão previamente detetada na citologia ${ }^{11,12}$.

As lesões intraepiteliais variam quanto à sua extensão na superfície do colo uterino, podendo o grau da lesão, por sua vez, variar ao longo da extensão da mesma, coexistindo simultaneamente áreas com lesão de baixo grau e áreas com lesões de alto grau ${ }^{13,19}$.

Para evitar um estudo inadequado da amostra histológica, é recomendada a realização de cortes histológicos seriados, bem como 0 estudo das margens excisadas, garantindo que as mesmas se encontram livres de lesão (fator imprescindível para garantir que a lesão foi totalmente removida) ${ }^{10,18}$.

No caso em estudo, foi efetuado este procedimento, permitindo verificar que uma das margens de excisão da conização intercetava áreas de lesão, indicando que a mesma não foi totalmente removida e, consequentemente, inadequadamente estudada. O diagnóstico final da conização foi de LSIL, apresentando áreas de CIN não graduável, discordando assim do diagnóstico citológico prévio. No entanto, uma vez que as margens se encontravam intercetadas pela lesão, pode ser levantada a hipótese de a lesão não estar totalmente representada na amostra.

\section{Falsos positivos/ sobrevalorização diagnóstica no exame citológico}

A sobrevalorização de critérios citológicos pode ser apontada como causa de discrepâncias cito-histológicas. Algumas causas de sobrevalorização e falsos positivos na citologia advêm de erros de diagnóstico diferencial. Exemplos de mimetizadores de 
lesões estão geralmente associados a casos de radiação ou outras terapêuticas, que podem simular lesões precursoras ou até carcinomas, ou ainda casos extremos de alterações reativas associadas à inflamação, reparação ou atrofia, que podem igualmente levar a erros de interpretação ${ }^{11}$.

No caso em estudo, foram observados achados citológicos atípicos que orientaram para um diagnóstico de carcinoma epidermóide. A contextualização destes achados num quadro de atrofia marcada justificou uma abordagem cautelosa, tendo sido ponderadas todas as hipóteses de diagnóstico.

O principal diagnóstico diferencial do carcinoma é o HSIL. O caso em estudo apresentava critérios citológicos comuns a ambos os diagnósticos, tais como marcadas anomalias nucleares, associadas a citoplasmas queratinizados, com algum pleomorfismo. Todavia, a existência de células queratinizadas, de extremidades citoplasmáticas alongadas, a presença de nucléolos proeminentes em alguns núcleos e o fundo hemático com detritos, muito sugestivo de diátese, foram as principais características citológicas que levaram à exclusão do diagnóstico de HSIL 20,21.

As alterações associadas à atrofia são também apontadas como diagnóstico diferencial de carcinoma. A atrofia benigna pode, pois, apresentar células isoladas, com núcleos grandes, alguma hipercromasia e citoplasmas densos eosinófilos ou oranginófilos (pseudo-paraqueratose). A presença de restos celulares amorfos ou inflamatórios, associada a núcleos nus, pode igualmente induzir a uma falsa interpretação de diátese tumoral20,21,22. No caso em estudo, a principal característica citológica que levou à exclusão deste diagnóstico foi a irregularidade das membranas nucleares e a anisocariose marcada.
A avaliação citológica de esfregaços pósmenopáusicos constitui, assim, um desafio, devido à forte relação entre 0 défice estrogénico e os achados atípicos verificados nas células pavimentosas. $\mathrm{Na}$ grande maioria dos casos, esta atipia aparente desaparece quando a paciente é submetida a terapêutica estrogénica, facilitando a separação entre casos com atipia e casos com achados normais de atrofia ${ }^{22}$.

No caso em estudo, a paciente foi submetida a terapêutica estrogénica, com vista a contornar eventuais pitfalls causados pela atrofia celular marcada. A citologia pós-terapia hormonal resultou num diagnóstico de LSIL. Foi ainda realizada nova biópsia de colo, tendo a mesma resultado num diagnóstico de LSIL, em contexto de marcada atrofia. Estes resultados remetem, uma vez mais, para a necessidade imperativa de follow-up destas pacientes. O novo diagnóstico citológico de LSIL (em oposição ao carcinoma ou mesmo HSIL), aponta novamente para a eventual sobrevalorização ocorrida no primeiro diagnóstico citológico, remetendo novamente, para a necessidade de abordagens prudentes na avaliação de citologias com padrões atróficos marcados.

Por fim, existe ainda referência na bibliografia, a duas outras eventuais justificações, para este tipo de discrepâncias. Por um lado, a existência de casos raros, em que a lesão regride no período de tempo decorrido entre o exame citológico e o estudo colposcópico e histológico10. Por outro lado, a possibilidade de existência de um carcinoma com outra localização que não o colo uterino (por exemplo, lesões localizadas na porção superior ou parede do canal vaginal ou mesmo localizações extrauterinas). Estes aspetos poderão originar falsos positivos e falsos negativos, quer na colposcopia, como na citologia ou ainda na histologia, não sendo de excluir estas hipóteses causais no caso aqui apresentado $^{14}$. 


\section{Exames complementares de diagnóstico}

No caso em estudo, foi realizada a deteção e tipificação de HPV, tendo a mesma revelado um resultado positivo para dois tipos de alto risco, estando em concordância com os achados cito-histológicos positivos.

O estudo imunohistoquímico de p16 e Ki67, na amostra de conização, poderia ser também uma mais-valia, para diferenciar entre a atrofia e HSIL. A marcação positiva para Ki67 na atrofia é muito limitada, enquanto que no HSIL a marcação é intensa. O p16 positivo é também muito útil na histologia, pois ajuda a identificar lesões ocultas ${ }^{21,23}$.

Os métodos complementares de diagnóstico surgem como uma solução de eficácia considerável no esclarecimento das dúvidas existentes nos casos com diagnósticos discrepantes, em que se torna difícil chegar a um consenso. A realização destes exames, menos subjetivos e mais precisos, permite aumentar a fiabilidade e confiança nos diagnósticos, diminuindo os impactos emocional e financeiro associados a eventuais erros $24,25,26$.

\section{CONCLUSÃO}

Considera-se pertinente o estudo deste caso, na medida em que apresenta um caso complexo, onde a correlação entre a colposcopia, a citologia e a histologia não foi atingida, bem como as possíveis causas para tal ocorrência.

Conclui-se que as causas mais comuns de falsos positivos na citologia são os erros de interpretação (sobrevalorização), enquanto as causas mais comuns de falsos negativos na histologia estão relacionadas com 0 procedimento de colheita e os erros de diagnóstico ${ }^{10,25}$.

O exame colposcópico, por si só, não constitui um método de diagnóstico fidedigno, por não ser suficientemente preciso. No entanto, não deixa de ter utilidade, direcionando o clínico, no que respeita o seguimento da utente e exames a efetuar ${ }^{18}$.

Salienta-se a importância da correlação entre os exames colposcópico, citológico e histológico, e outros exames complementares de diagnóstico. A comunicação entre os profissionais de saúde das diversas áreas envolvidas no diagnóstico de lesões cervicais torna-se, evidentemente, um ponto crucial na obtenção de um diagnóstico efetivo e fidedigno, permitindo, desta forma, a adoção de medidas de follow-up adequadas ${ }^{18}$.

\section{Agradecimentos}

Agradecemos à Dra. Maria Fernanda Cunha e Dra. Cristina Amado, pela disponibilização do caso e pelos esclarecimentos prestados, nomeadamente nos diagnósticos histológicos apresentados ao longo do estudo.

A elaboração deste estudo também não teria sido possível sem o apoio incondicional do Dr. Jorge Pereira e do Dr. Horácio Scigliano.

Um agradecimento especial vai também para a Técnica Cidália Teixeira e para as Técnicas Joana Pereira e Helena Andrez, cuja ajuda no processo de recolha de informações e fotografias do caso, foi também fundamental.

\section{REFERÊNCIAS BIBLIOGRÁFICAS}

1. Patel M, Pandya A. Cervical pap smear study and its utility in cancer screening, to specify the strategy for cervical cancer control. National Journal of Community Medicine. 2011;2(1):4951.

2. Arbeit J, Howley P. Chronic estrogen induced cervical and vaginal squamous carcinogenesis in human papillomavirus type 16 transgenic mice. Medical sciences. 1996;93:2930-2935. 
3. McQuillan G, Kruszon-Moran D, Markowitz L, Unger E, Paulose-Ram R. Prevalence of HPV in Adults Aged 18-69: United States, 20112014. Centers for Disease Control and Prevention of the National Center for Health Statistics. 2017:280.

4. Kietpeerakool C, Scrisomboon J. How can the overtreatment rate of "see and treat" approach be reduced in women with high-grade squamous intraepithelial lesion on cervical cytology. Asian Pacific Journal of Cancer Prevention. 2007;8:206-208.

5. Cantor S, Turanzas M, Accuracy of colposcopy in the diagnostic setting compared with the screening setting. American College of Obstetricians and Gynecologists. 2008;111(1):7-14.

6. Colgan T, Woodhouse S, Reparative changes and the false-positive/false-negative papanicolaou cytology. Archives of Pathology. 2001;125:134-139.

7. Saad R, Dabbs D, Clinical significance of cytology diagnosis of atypical squamous cells, cannot exclude high grade, in perimenopausal and postmenopausal women. American Society for Clinical Pathology. 2006;126:381-388.

8. Nayar R, Wilbur DC. The Bethesda System for Reporting Cervical Cytology - Definitions, Criteria and Explanatory notes. $3^{\text {a }}$ ed. New York: Springer; 2014. Chapter 5, Epithelial Abnormalities: Squamous; p. 99-116.

9. Koss LG. Diagnostic Cytopthology and its histopathology bases. Vol 1. $5^{\underline{a}}$ ed. JB Lippincott Company; 2006. Chapter 11, Epidermoid Carcinoma of the Uterine Cervix and Related Precancerous Lesions; p. 371428.

10. Joseph MG, Cragg, F, Wright C, Kontozoglou TE, Downing P, Marks FR. Cyto-Histological Correlates in a Colposcopic Clinic: A I-Year Prospective Study. Diagnostic Cytopathology. 1991;7:477-481.

11. Dodd LG, Sneige N, Villarreal $Y$, Fanning C, Staerkel GA, Caraway NP, Silva EG, Katz RL. Quality-Assurance Study of Simultaneously Sampled, Non-Correlating Cervical Cytology and Biopsies. Diagnostic Cytopathology. 1993;9:138-144.

12. DiBonito L, Falconieri G, Tomasic G, Colauffi I, Bonifacio D, Dudine S. Cervical Cytopathology:
An Evaluation of Its Accuracy Based on Cytohistologic Comparison. Cancer Cytopathology. 1993;72:3002-3006.

13. Heatley MK, Bury JP. The correlation between the grade of dyskaryosis on cervical smear, grade of cervical intraepithelial neoplasia (CIN) on punch biopsy and the final histological diagnosis on cone biopsies of the cervix. Cytopathology. 1998;9:93-99.

14. Lonky NM, Sadeghi M, Tsadik GM, Petitti D. The clinical significance of the poor correlation of cervical dysplasia and cervical malignancy with referral cytologic results. American Journal of Obstetrics and Gynecology. 1999;181: 560566.

15. Szurkus DC, Harrison TA. Loop excision for highgrade squamous intraepithelial lesion on cytology: Correlation with colposcopic and histologic findings. American Journal of Obstetrics and Gynecology. 2003;188:11801182.

16. Saha R, Thapa M. Correlation of cervical cytology with cervical histology. Kathmandu University Medical Journal. 2005;3(3,11):222-224.

17. Kabaca C, Sariibrahim B, Keleli I, Karateke A, Cesur S, Cetiner $\mathrm{H}$. The importance of immediate verification of a cervical cytological abnormality with histology. Indian Journal of Cancer. 2013;50:292-296.

18. Milenkovic V, Sparic R, Dotlic J, Tulic L, Mirkovic L, Milenkovic S, Atanackovic J. Reliability and relationship of colposcopical, cytological and histopathological findings in the diagnostic process. Clinic for Gynaecology and Obstetrics. 2012; 69(10): 869-873.

19. Zuchna C, Hager M, Tringler B, Georgoulopoulos A, Ciresa-Koenig A, Volgger B, Widschwendter A, Satudach A. Diagnostic accuracy of guided cervical biopsies: a prospective multicenter study comparing the histopathology of simultaneous biopsy and cone specimen. American Journal of Obstetrics and Gynecology. 2010;203:321.

20. Cibas ES, Ducatman BS. Cytology - Diagnostic principles and clinical correlates. $2^{\underline{a}}$ ed. Philadelphia: Saunders Elsevier; 2003. Chapter 1, Cervical and vaginal cytology; p. 31-38.

21. Nucci MR, Oliva E, Goldblum, JR. Gynecologic Pathology - A volume in the series. 
Foundations in Diagnostic Pathology. $1^{\mathrm{a}}$ ed. Churchil Livingston - Elsevier; 2009. 667 p.

22. Abati A, Jaffurs W, Wilder AM. Squamous Atypia in the Atrophic Cervical Vaginal Smear: A New Look at an Old Problem. Cancer Cytopathology. 1998;84(4)218-225.

23. Rudiger $\mathrm{K}$, Benner $\mathrm{A}$, Friedrich $\mathrm{T}$, Ridder $\mathrm{T}$, Herrington S, Jenkins D, Kurman RJ, Schmidt D, Stoler M, Doeberitz MK. p16INK4a Immunohistochemistry Improves Interobserver Agreement in the Diagnosis of Cervical Intraepithelial Neoplasia. The American Journal of Surgical Pathology. 2002;26(11):1389-1399.

24. Jones BA, Novis DA. Cervical Biopsy-Cytology Correlation: A College of American Pathologists Q-Probes Study of 22439 Correlations in 348 Laboratories. Archives of Pathology Lab Med. 1996;120:523-531.

25. Joste NE, Crum CP, Cibas ES. Cytologic/Histologic Correlation for Quality Control in Cervicovaginal Cytology: Experience With 1,582 Paired Cases. American Journal of Clinical Pathology. 1995;103(1)32-34.

26. Medley G, Surtees VM. Squamous Atypia in the Atrophic Cervical Vaginal Smear - A Plea for a More Painstaking Old Style Look versus a New Look at the Old Problem. Cancer Cytopathology. 1998;84(4)200-201. 